\title{
New Condition of Stabilization of Uncertain Continuous Takagi-Sugeno Fuzzy System based on Fuzzy Lyapunov Function
}

\author{
Yassine Manai \\ LA.R.A. Automatique, National School of Engineer of Tunis, El-Manar University, Tunis, Tunisia \\ Email:yacine.manai@gmail.com \\ Mohamed Benrejeb \\ LA.R.A. Automatique, National School of Engineer of Tunis, El-Manar University, Tunis, Tunisia \\ Email: Mohamed.benrejeb@enit.rnu.tn
}

\begin{abstract}
This paper deals with the stabilization of TakagiSugeno fuzzy models. Using non-quadratic Lyapunov function, new sufficient stabilization criteria with PDC controller are established in terms of Linear Matrix Inequality. Finally, a stabilization condition for uncertain system is given.
\end{abstract}

Index Terms - Takagi-Sugeno fuzzy system, uncertain system, Linear Matrix Inequalities LMIs, Fuzzy Lyapunov Function, Parallel Distributed Compensation PDC.

\section{INTRODUCTION}

Fuzzy control systems have experienced a big growth of industrial applications in the recent decades, because of their reliability and effectiveness. Many researches are investigated on the Takagi-Sugeno models [1-2] which can combine the flexible fuzzy logic theory and rigorous mathematical theory into a unified framework. Thus, it becomes a powerful tool in approximating a complex nonlinear system.

Two classes of Lyapunov functions are used to analysis these systems: quadratic Lyapunov functions and non-quadratic Lyapunov ones which are less conservative than first class. Many researches are investigated with non-quadratic Lyapunov functions [1-9].

In this paper, a new stability conditions for Takagi Sugeno uncertain fuzzy models based on the use of fuzzy Lyapunov function are presented. This criterion is expressed in terms of Linear Matrix Inequalities (LMIs) which can be efficiently solved by using various convex optimization algorithms [10]. The presented method is les conservative than existing results.

The organization of the paper is as follows. In section 2, we present the system description and problem formulation and we give some preliminaries which are needed to derive results. Section 3 will be concerned to stability analysis for T-S fuzzy systems. Section 4 concerns the proposed approach to stabilize a T-S fuzzy system with Parallel Distributed Compensation (PDC). Next, a new stabilization condition for uncertain system is given. Finally section 6 makes conclusion.
Notation: Throughout this paper, a real symmetric matrix $S>0$ denotes $S$ being a positive definite matrix. The superscript " $T$ " is used for the transpose of a matrix.

\section{SYSTEM DESCRIPTION AND PRELIMINARIES}

Consider an uncertain T-S fuzzy continuous model for a nonlinear systemas follows:

$$
\begin{gathered}
\text { IF } z_{1}(t) \text { is } M_{i 1} \text { and ...and } z_{p}(t) \text { is } M_{i p} \\
\text { THEN } \dot{x}(t)=\left(A_{i}+\Delta A_{i}\right) x(t)+\left(B_{i}+\Delta B_{i}\right) u(t) \\
\quad i=1, \ldots, r
\end{gathered}
$$

where $M_{i j}(i=1,2, \ldots, r, j=1,2, \ldots, p)$ is the fuzzy set and $\mathrm{r}$ is the number of model rules; $x(t) \in \mathfrak{R}^{n}$ is the state vector, $u(t) \in \mathfrak{R}^{m}$ is the input vector, $A_{i} \in \mathfrak{R}^{n \times n}$, $B_{i} \in \mathfrak{R}^{n \times m}$, and $z_{1}(t), \ldots, z_{p}(t)$ are known premise variables. $\Delta A_{i}$ and $\Delta B_{i}$ are time-varying matrices representing parametric uncertainties in the plant model. These uncertainties are admissibly norm-bounded and structured.

The final outputs of the fuzzy systems are:

$$
\begin{aligned}
& \dot{x}(t)=\sum_{i=1}^{r} h_{i}(z(t))\left\{\left(A_{i}+\Delta A_{i}\right) x(t)+\left(B_{i}+\Delta B_{i}\right) u(t)\right\} \\
& \text { where } \\
& z(t)=\left[z_{1}(t) z_{2}(t) \ldots z_{p}(t)\right] \\
& h_{i}(z(t))=w_{i}(z(t)) / \sum_{i=1}^{r} w_{i}(z(t)) \\
& w_{i}(z(t))=\prod_{j=1}^{p} M_{i j}\left(z_{j}(t)\right) \text { for all t. }
\end{aligned}
$$

The term $M_{i 1}\left(z_{j}(t)\right)$ is the grade of membership of $z_{j}(t)$ in $M_{i 1}$ 
Since $\left\{\begin{array}{l}\sum_{i=1}^{r} w_{i}(z(t)) \succ 0 \\ w_{i}(z(t)) \geq 0,\end{array}\right.$
we have $\left\{\begin{array}{l}\sum_{i=1}^{r} h_{i}(z(t))=1,2, \ldots, r \\ h_{i}(z(t)) \geq 0,\end{array} \quad\right.$ for all t.

The time derivative of premise membership functions is given by:

$\dot{h}_{i}(z(t))=\frac{\partial h_{i}}{\partial z(t)} \cdot \frac{\partial z(t)}{\partial x(t)} \cdot \frac{d x(t)}{d t}=\sum_{l=1}^{s} v_{i l} \xi_{i l} \times \frac{d x(t)}{d t}$

We have the following property:

$$
\sum_{k=1}^{r} \dot{h}_{k}(z(t))=0
$$

The PDC fuzzy controller is represented by

$$
u(t)=-\frac{\sum_{i=1}^{r} w_{i}(z(t)) F_{i} x(t)}{\sum_{i=1}^{r} w_{i}(z(t))}=-\sum_{i=1}^{r} h_{i}(z(t)) F_{i} x(t) \text { (5) }
$$

The fuzzy controller design is to determine the local feedback gains $F_{i}$ in the consequent parts.

The open-loop systemis given by the equation (6)

$$
\dot{x}(t)=\sum_{i=1}^{r} h_{i}(z(t))\left(A_{i}+\Delta A_{i}\right) x(t)
$$

By substituting (5) into (2), the closed-loop fuzzy systemcan be represented as:

$$
\begin{gathered}
\dot{x}(t)=\sum_{i=1}^{r} \sum_{j=1}^{r} h_{i}(z(t)) h_{j}(z(t))\left\{A_{\Delta i}-B_{\Delta i} F_{j}\right\} x(t) \\
\text { where } A_{\Delta i}=A_{i}+\Delta A_{i} \text { and } B_{\Delta i}=B_{i}+\Delta B_{i}
\end{gathered}
$$

\section{Assumption 1}

The time derivative of the premises membership function is upper bounded such that $\left|\dot{h}_{k}\right| \leq \phi_{k}$, for $k=1, \ldots, r$, where, $\phi_{k}, k=1, \ldots, r$ are given positive constants.

\section{Assumption 2}

The matrices denote the uncertainties in the system and take the form of

$$
\left[\begin{array}{cc}
\Delta A_{i} & \Delta B_{i}
\end{array}\right]=D F(t)\left[\begin{array}{ll}
E_{A_{i}} & E_{B_{i}}
\end{array}\right]
$$

where $D, E_{A_{i}}$ and $E_{B_{i}}$ are known constant matrices and $F(t)$ is an unknown matrix function satisfying:

$$
F^{T}(t) F(t) \leq I, \forall t
$$

where $I$ is an appropriately dimensioned identity matrix.

\section{Lemma 1 (Boyd et al. Schur complement [6])}

Given constant matrices $\Omega_{1}, \Omega_{2}$ and $\Omega_{3}$ with appropriate dimensions, where $\Omega_{1}=\Omega_{1}^{T}$ and $\Omega_{2}=\Omega_{2}^{T}$, then

$$
\begin{aligned}
\Omega_{1}+\Omega_{3}^{T} \Omega_{2}^{-1} \Omega_{3} & \prec 0 \\
& \text { if and only if }
\end{aligned}
$$

$$
\left[\begin{array}{cc}
\Omega_{1} & \Omega_{3}^{T} \\
* & -\Omega_{2}
\end{array}\right] \text { p } 0 \text { or }\left[\begin{array}{cc}
-\Omega_{2} & \Omega_{3} \\
* & \Omega_{1}
\end{array}\right] \text { p } 0
$$

\section{Lemma 2 (Peterson and Hollot [8])}

Let $Q=Q^{T}, H, E \quad$ and $\quad F(t) \quad$ satisfying $F^{T}(t) F(t) \leq I$ are appropriately dimensional matrices then the following inequality

$$
Q+H F(t) E+E^{T} F^{T}(t) H^{T} \text { p } 0
$$

is true, if and only if the following inequality holds for any $\varepsilon \mathrm{f} 0$

$$
Q+\varepsilon^{-1} H H^{T}+\varepsilon E^{T} E \mathrm{p} 0
$$

\section{BASIC STABILITY CONDITIONS}

Consider the open-loop system(8).

$$
\dot{x}(t)=\sum_{i=1}^{r} h_{i}(z(t)) A_{i} x(t)
$$

This section gives a new condition for stability of the unforced T-S fuzzy system by using the Lyapunov theory.

\section{Theorem 1 [11]}

Under assumption 1 and for $0 \leq \varepsilon \leq 1$, the Takagi Sugeno fuzzy system (8) is stable if there exist positive definite symmetric matrices $P_{k}, k=1,2, \ldots, r$, matrix $R=R^{T}$ such that the following LMIs hold.

$$
\begin{aligned}
& P_{k}+R \succ 0, \quad k \in\{1, \ldots, r\} \\
& P_{j}+\mu R \succ 0, \quad j \in\{1, \ldots, r\}
\end{aligned}
$$

$$
\begin{aligned}
P_{\phi} & +\frac{1}{2}\left\{A_{i}^{T}\left(P_{j}+\mu R\right)+\left(P_{j}+\mu R\right) A_{i}\right. \\
& \left.+A_{j}^{T}\left(P_{i}+\mu R\right)+\left(P_{i}+\mu R\right) A_{j}\right\} \prec 0, \quad i \leq j
\end{aligned}
$$

where $i, j=1,2, \ldots, r$ and $P_{\phi}=\sum_{k=1}^{r} \phi_{k}\left(P_{k}+R\right)$ and $\mu=1-\varepsilon$

\section{STABILIZATION WITH PDC CONTROLLER}

Consider the closed-loop system without uncertainties which can be rewritten as 


$$
\begin{aligned}
& \dot{x}(t)=\sum_{i=1}^{r} h_{i}(z(t)) h_{i}(z(t)) G_{i i} x(t) \\
& +2 \sum_{i=1}^{r} \sum_{i \prec j} h_{i}(z(t)) h_{j}(z(t))\left\{\frac{G_{i j}+G_{j i}}{2}\right\} x(t),
\end{aligned}
$$

where

$$
G_{i j}=A_{i}-B_{i} F_{j} \text { and } G_{i i}=A_{i}-B_{i} F_{i} .
$$

In this section we define a fuzzy Lyapunov function and then consider stability conditions.

\section{Theorem 2}

Under assumption 1 and for given $0 \leq \varepsilon \leq 1$, the Takagi-Sugeno system (12) is stable if there exist positive definite symmetric matrices $P_{k}, k=1,2, \ldots, r$, and $R$, matrices $F_{1}, \ldots, F_{r}$ such that the following LMIs hols.

$$
\begin{array}{ll}
P_{k}+R \succ 0, & k \in\{1, \ldots, r\} \\
P_{j}+\mu R \geq 0, & j=1,2, \ldots, r
\end{array}
$$

$P_{\phi}+\left\{G_{i i}^{T}\left(P_{k}+\mu R\right)+\left(P_{k}+\mu R\right) G_{i i}\right\} \prec 0$,

$i, k \in\{1, \ldots, r\}$

$$
\left\{\frac{G_{i j}+G_{j i}}{2}\right\}^{T}\left(P_{k}+\mu R\right)+\left(P_{k}+\mu R\right)\left\{\frac{G_{i j}+G_{j i}}{2}\right\} \prec 0,
$$

for $i, j, k=1,2, \ldots, r$ such that $i \prec j$

where

$$
G_{i j}=A_{i}-B_{i} F_{j}, G_{i i}=A_{i}-B_{i} F_{i}, \mu=1-\varepsilon,
$$$$
\text { and } P_{\phi}=\sum_{k=1}^{r} \phi_{k}\left(P_{k}+R\right)
$$

Proof form:

Let consider the Lyapunov function in the following

$$
V(x(t))=\sum_{k=1}^{r} h_{k}(z(t)) \cdot V_{k}(x(t))
$$

with

$$
V_{k}(x(t))=x^{T}(t)\left(P_{k}+\mu R\right) x(t), \quad k=1,2, \ldots, r
$$

where

$$
\begin{aligned}
& P_{k}=P_{k}^{T}, R=R^{T}, 0 \leq \varepsilon \leq 1, \mu=1-\varepsilon, \\
& \text { and }\left(P_{k}+\mu R\right) \geq 0, \quad k=1,2, \ldots, r
\end{aligned}
$$

The time derivative of $V(x(t))$ with respect to $t$ along the trajectory of the system (12) is given by:

$$
\dot{V}(x(t))=\sum_{k=1}^{r} \dot{h_{k}}(z(t)) V_{k}(x(t))+\sum_{k=1}^{r} h_{k}(z(t)) \dot{V_{k}}(x(t))
$$

$$
\begin{array}{r}
\dot{V}(x(t))=x^{T}(t)\left(\sum_{k=1}^{r} \dot{h}_{k}(z(t))\left(P_{k}+\mu R\right)\right) x(t) \\
+\dot{x}^{T}(t)\left(\sum_{k=1}^{r} h_{k}(z(t))\left(P_{k}+\mu R\right)\right) x(t) \\
+x^{T}(t)\left(\sum_{k=1}^{r} h_{k}(z(t))\left(P_{k}+\mu R\right)\right) \dot{x}(t)
\end{array}
$$

By substituting (12) into (19), we obtain, $\dot{V}(x(t))=\Upsilon_{1}(x, z)+\Upsilon_{2}(x, z)+\Upsilon_{3}(x, z)(20)$

where

$$
\begin{aligned}
& \Upsilon_{1}(x, z)=x^{T}(t)\left(\sum_{k=1}^{r} \dot{h_{k}}(z(t)) \cdot\left(P_{k}+\mu R\right)\right) x(t)(21) \\
& \Upsilon_{2}(x, z)=x^{T}(t) \sum_{k=1}^{r} \sum_{i=1}^{r} h_{k}(z(t)) h_{i}^{2}(z(t)) \\
& \quad \times\left\{G_{i i}^{T}\left(P_{k}+\mu R\right)+\left(P_{k}+\mu R\right) G_{i i}\right\} x(t) \\
& \Upsilon_{3}(x, z)=x(t)^{T} \sum_{k=1}^{r} \sum_{i=1}^{r} \sum_{i \prec j} h_{k}(z(t)) h_{i}(z(t)) h_{j}(z(t)) \\
& \left.\times\left\{\left(\frac{G_{i j}+G_{j i}}{2}\right)^{T}\left(P_{k}+\mu R\right)+\left(P_{k}+\mu R\right)\left(\frac{G_{i j}+G_{j i}}{2}\right)\right\}\right] x(t)
\end{aligned}
$$

Then, based on assumption 1, an upper bound of $\Upsilon_{1}(x, z)$ obtained as:

$$
\Upsilon_{1}(x, z) \leq \sum_{k=1}^{r} \phi_{k} \cdot x(t)^{T}\left(P_{k}+\mu R\right) x(t)
$$

Based on (4), it follows that $\sum_{k=1}^{r} \dot{h}_{k}(z(t)) \varepsilon R=\bar{R}=0$ where $\mathrm{R}$ is any symmetric matrix of proper dimension.

Adding $\bar{R}$ to (24), then

$$
\Upsilon_{1}(x, z) \leq \sum_{k=1}^{r} \phi_{k} \cdot x(t)^{T}\left(P_{k}+R\right) x(t)
$$

Then,

$$
\begin{aligned}
\dot{V}(x(t)) \leq & \sum_{k=1}^{r} \phi_{k} x^{T}(t)\left(P_{k}+R\right) x(t) \\
& +\Upsilon_{2}(x, z)+\Upsilon_{3}(x, z)
\end{aligned}
$$

If (15) and (16) holds, the time derivative of the fuzzy Lyapunov function is negative. Consequently, we have

The equation (18) can be rewritten as, 


$$
\begin{aligned}
& \dot{V}(x(t)) \leq x^{T}(t)\left(\sum_{\mathrm{k}=1}^{\mathrm{r}} \sum_{\mathrm{i}=1}^{\mathrm{r}} h_{k}(z(t)) h_{i}^{2}(z(t))\right. \\
& \times\left\{\left(G_{i i}^{T}\left(P_{k}+\mu R\right)+\left(P_{k}+\mu R\right) G_{i i}\right)\right\} \\
& +\sum_{k=1}^{r} \phi_{k}\left(\left(P_{k}+R\right)\right)+\sum_{\mathrm{k}=1}^{\mathrm{r}} \sum_{\mathrm{i}=1}^{\mathrm{r}} \sum_{i \prec j} h_{k}(z(t)) h_{i}(z(t)) h_{j}(z(t)) \\
& \left.\times\left(\left\{\frac{G_{i j}+G_{j i}}{2}\right\}^{T}\left(P_{k}+\mu R\right)+\left(P_{k}+\mu R\right)\left\{\frac{G_{i j}+G_{j i}}{2}\right\}\right)\right) x(t) \\
& \prec 0
\end{aligned}
$$

and the closed loop fuzzy system (12) is stable. This is complete the proof.

\section{Robust STABILITY CONDITION WITH PDC CONTRO LLER}

Consider the closed-loop system (7). A sufficient robust stability condition is given follow.

\section{Theorem 3}

Under assumption 1, and assumption 2 and for given $0 \leq \varepsilon \leq 1$, the Takagi-Sugeno system (7) is stable if there exist positive definite symmetric matrices $P_{k}, k=1,2, \ldots, r$, and $R$, matrices $F_{1}, \ldots, F_{r}$ such that the following LMIs hols.

$$
\begin{aligned}
& P_{k}+R \succ 0, \quad k \in\{1, \ldots, r\} \\
& P_{j}+\mu R \geq 0, \quad j=1,2, \ldots, r \\
& {\left[\begin{array}{ccc}
\Phi_{1} & \left(P_{k}+\mu R\right) D_{a i} & \left(P_{k}+\mu R\right) D_{b i} \\
* & -\lambda I & 0 \\
* & * & -\lambda I
\end{array}\right] \prec 0} \\
& i, k \in\{1, \ldots, r\} \\
& \text { with } \\
& \Phi_{1}=P_{\phi}+G_{i i}^{T}\left(P_{k}+\mu R\right)+\left(P_{k}+\mu R\right) G_{i i} \\
& +\lambda\left(P_{k}+\mu R\right)\left[E_{a i}^{T} E_{a i}+\left(E_{b i} F_{i}\right)^{T} E_{b i} F_{i}\right] \\
& {\left[\begin{array}{ccc}
\Phi_{2} & \left(P_{k}+\mu R\right)\left(D_{a i}+D_{a j}\right) & \left(P_{k}+\mu R\right)\left(D_{b i}+D_{b j}\right) \\
* & -\lambda I & 0 \\
* & * & -\lambda I
\end{array}\right] \prec 0}
\end{aligned}
$$

for $i, j, k=1,2, \ldots, r$ such that $i \prec j$

with

$$
\begin{aligned}
\Phi_{2} & =\left(\frac{G_{i j}+G_{j i}}{2}\right)^{T}\left(P_{k}+\mu R\right)+\left(P_{k}+\mu R\right)\left(\frac{G_{i j}+G_{j i}}{2}\right) \\
& +\lambda\left(P_{k}+\mu R\right)\left[\left(E_{a i}+E_{a j}\right)^{T}\left(E_{a i}+E_{a j}\right)\right. \\
& \left.+\left(E_{b i} F_{j}+E_{b j} F_{i}\right)^{T}\left(E_{b i} F_{j}+E_{b j} F_{i}\right)^{T}\right]
\end{aligned}
$$

where

$$
G_{i j}=A_{i}-B_{i} F_{j} \quad, \quad G_{i i}=A_{i}-B_{i} F_{i} \quad,
$$

$$
\mu=1-\varepsilon, \text { and } P_{\phi}=\sum_{k=1}^{r} \phi_{k}\left(P_{k}+R\right)
$$

\section{Proof} form:

Let consider the Lyapunov function in the following

$$
V(x(t))=\sum_{k=1}^{r} h_{k}(z(t)) \cdot V_{k}(x(t))(30)
$$

with

$$
V_{k}(x(t))=x^{T}(t)\left(P_{k}+\mu R\right) x(t), k=1,2, \ldots, r
$$

where

$$
\begin{aligned}
& P_{k}=P_{k}^{T}, R=R^{T}, 0 \leq \varepsilon \leq 1, \mu=1-\varepsilon, \\
& \text { and }\left(P_{k}+\mu R\right) \geq 0, \quad k=1,2, \ldots, r
\end{aligned}
$$

The time derivative of $V(x(t))$ with respect to $t$ along the trajectory of the system (12) is given by:

$\dot{V}(x(t))=\sum_{k=1}^{r} \dot{h_{k}}(z(t)) V_{k}(x(t))+\sum_{k=1}^{r} h_{k}(z(t)) \dot{V_{k}}(x(t))$

The equation (31) can be rewritten as,

$$
\begin{array}{r}
\dot{V}(x(t))=x^{T}(t)\left(\sum_{k=1}^{r} \dot{h}_{k}(z(t))\left(P_{k}+\mu R\right)\right) x(t) \\
+\dot{x}^{T}(t)\left(\sum_{k=1}^{r} h_{k}(z(t))\left(P_{k}+\mu R\right)\right) x(t) \\
+x^{T}(t)\left(\sum_{k=1}^{r} h_{k}(z(t))\left(P_{k}+\mu R\right)\right) \dot{x}(t)
\end{array}
$$

By substituting (7) into (32), we obtain,

$$
\dot{V}(x(t))=\Upsilon_{1}(x, z)+\Upsilon_{2}(x, z)+\Upsilon_{3}(x, z)
$$

where

$$
\begin{aligned}
& \Upsilon_{1}(x, z)=x^{T}(t)\left(\sum_{k=1}^{r} \dot{h}_{k}(z(t)) \cdot\left(P_{k}+\mu R\right)\right) x(t) \\
& \quad \Upsilon_{2}(x, z)=x^{T}(t) \sum_{k=1}^{r} \sum_{i=1}^{r} h_{k}(z(t)) h_{i}^{2}(z(t)) \\
& \quad \times\left\{G_{i i}^{T}\left(P_{k}+\mu R\right)+\left(P_{k}+\mu R\right) G_{i i}\right\} x(t) \\
& +x^{T}(t) \sum_{k=1}^{r} \sum_{i=1}^{r} h_{k}(z(t)) h_{i}^{2}(z(t)) \\
& \quad \times\left\{\left(\left[\begin{array}{ll}
D_{a i} & D_{b i}
\end{array}\right]\left[\begin{array}{cc}
\Delta_{a i} & 0 \\
0 & \Delta_{b i}
\end{array}\right]\left[\begin{array}{l}
E_{a i} \\
-E_{b i} F_{i}
\end{array}\right]\right)^{T}\left(P_{k}+\mu R\right)\right. \\
& \left.\quad+\left(P_{k}+\mu R\right)\left(\left[\begin{array}{ll}
D_{a i} & D_{b i}
\end{array}\right]\left[\begin{array}{cc}
\Delta_{a i} & 0 \\
0 & \Delta_{b i}
\end{array}\right]\left[\begin{array}{l}
E_{a i} \\
-E_{b i} F_{i}
\end{array}\right]\right)\right\} x(t)
\end{aligned}
$$




$$
\begin{aligned}
& \Upsilon_{3}(x, z)=x(t)^{T} \sum_{k=1}^{r} \sum_{i=1}^{r} \sum_{i \prec j} h_{k}(z(t)) h_{i}(z(t)) h_{j}(z(t)) \\
& \left.\times\left\{\left(\frac{G_{i j}+G_{j i}}{2}\right)^{T}\left(P_{k}+\mu R\right)+\left(P_{k}+\mu R\right)\left(\frac{\left.G_{i j}+G_{j i}\right)}{2}\right)\right\}\right] x(t) \\
& +x(t)^{T} \sum_{k=1}^{r} \sum_{i=1}^{r} \sum_{i<j} h_{k}(z(t)) h_{i}(z(t)) h_{j}(z(t)) \\
& \times\left\{\left(\left[\begin{array}{ll}
D_{a i} & D_{b i}
\end{array}\right]\left[\begin{array}{cc}
\Delta_{a i} & 0 \\
0 & \Delta_{b i}
\end{array}\right]\left[\begin{array}{ll}
E_{a i} \\
-E_{b i} F_{j}
\end{array}\right]\right)^{T}\left(P_{k}+\mu R\right)\right. \\
& \left.+\left(P_{k}+\mu R\right)\left(\left[\begin{array}{cc}
D_{a i} & D_{b i}
\end{array}\right]\left[\begin{array}{cc}
\Delta_{a i} & 0 \\
0 & \Delta_{b i}
\end{array}\right]\left[\begin{array}{l}
E_{a i} \\
-E_{b i} F_{j}
\end{array}\right]\right)\right\} x(t) \\
& +x(t)^{T} \sum_{k=1}^{r} \sum_{i=1}^{r} \sum_{i<j} h_{k}(z(t)) h_{i}(z(t)) h_{j}(z(t)) \\
& \times\left\{\left(\left[\begin{array}{ll}
D_{a j} & D_{b j}
\end{array}\right]\left[\begin{array}{cc}
\Delta_{a j} & 0 \\
0 & \Delta_{b j}
\end{array}\right]\left[\begin{array}{c}
E_{a j} \\
-E_{b j} F_{i}
\end{array}\right]\right)^{T}\left(P_{k}+\mu R\right)\right. \\
& \left.+\left(P_{k}+\mu R\right)\left(\left[\begin{array}{cc}
D_{a j} & D_{b j}
\end{array}\right]\left[\begin{array}{cc}
\Delta_{a j} & 0 \\
0 & \Delta_{b j}
\end{array}\right]\left[\begin{array}{l}
E_{a j} \\
-E_{b j} F_{i}
\end{array}\right]\right)\right\} x(t)
\end{aligned}
$$

Then, based on assumption 1, an upper bound of $\Upsilon_{1}(x, z)$ obtained as:

$$
\Upsilon_{1}(x, z) \leq \sum_{k=1}^{r} \phi_{k} \cdot x(t)^{T}\left(P_{k}+\mu R\right) x(t)
$$

Based on (4), it follows that $\sum_{k=1}^{r} \dot{h}_{k}(z(t)) \varepsilon R=\bar{R}=0$ where $\mathrm{R}$ is any symmetric matrix of proper dimension.

Adding $\bar{R}$ to (34), then

$$
\Upsilon_{1}(x, z) \leq \sum_{k=1}^{r} \phi_{k} \cdot x(t)^{T}\left(P_{k}+R\right) x(t)
$$

Then,

$$
\dot{V}(x(t)) \leq \sum_{k=1}^{r} \phi_{k} x^{T}(t)\left(P_{k}+R\right) x(t)+\Upsilon_{2}(x, z)+\Upsilon_{3}(x, z)
$$

If

$$
\sum_{k=1}^{r} \phi_{k}\left(P_{k}+R\right)+G_{i i}^{T}\left(P_{k}+\mu R\right)+\left(P_{k}+\mu R\right) G_{i i}
$$$$
+\left\{\left(\left[\begin{array}{ll}
D_{a i} & D_{b i}
\end{array}\right]\left[\begin{array}{cc}
\Delta_{a i} & 0 \\
0 & \Delta_{b i}
\end{array}\right]\left[\begin{array}{l}
E_{a i} \\
-E_{b i} F_{i}
\end{array}\right]\right)^{T}\left(P_{k}+\mu R\right)\right.
$$$$
\left.+\left(P_{k}+\mu R\right)\left(\left[\begin{array}{ll}
D_{a i} & D_{b i}
\end{array}\right]\left[\begin{array}{cc}
\Delta_{a i} & 0 \\
0 & \Delta_{b i}
\end{array}\right]\left[\begin{array}{l}
E_{a i} \\
-E_{b i} F_{i}
\end{array}\right]\right)\right\} \prec 0
$$

Then, based on Lemma 2, an upper bound of $\Upsilon_{1}(x, z)$ obtained as:

$$
\begin{aligned}
& \sum_{k=1}^{r} \phi_{k}\left(P_{k}+R\right)+G_{i i}^{T}\left(P_{k}+\mu R\right)+\left(P_{k}+\mu R\right) G_{i i} \\
& +\lambda^{-1}\left(P_{k}+\mu R\right)\left[\begin{array}{ll}
D_{a i} & D_{b i}
\end{array}\right]\left[\begin{array}{c}
D_{a i}^{T} \\
D_{b i}^{T}
\end{array}\right] \\
& +\lambda\left[\begin{array}{ll}
E_{a i}^{T} & -\left(E_{b i} F_{i}\right)^{T}
\end{array}\right]\left[\begin{array}{l}
E_{a i} \\
-E_{b i} F_{i}
\end{array}\right]\left(P_{k}+\mu R\right) \prec 0
\end{aligned}
$$

by Schur complement, we obtain,

$$
\left[\begin{array}{ccc}
\Phi_{1} & \left(P_{k}+\mu R\right) D_{a i} & \left(P_{k}+\mu R\right) D_{b i} \\
* & -\lambda I & 0 \\
* & * & -\lambda I
\end{array}\right] \prec 0
$$

with

$$
\begin{aligned}
& \Phi_{1}=P_{\phi}+G_{i i}^{T}\left(P_{k}+\mu R\right)+\left(P_{k}+\mu R\right) G_{i i} \\
& +\lambda\left(P_{k}+\mu R\right)\left[E_{a i}^{T} E_{a i}+\left(E_{b i} F_{i}\right)^{T} E_{b i} F_{i}\right] \\
& \left\{\left(\frac{G_{i j}+G_{j i}}{2}\right)^{T}\left(P_{k}+\mu R\right)+\left(P_{k}+\mu R\right)\left(\frac{G_{i j}+G_{j i}}{2}\right)\right\} \\
& +\left\{\left(\left[\begin{array}{ll}
D_{a i}+D_{a j} & D_{b i}+D_{b j}
\end{array}\right]\left[\begin{array}{cc}
\Delta_{a i}+\Delta_{a j} & 0 \\
0 & \Delta_{b i}+\Delta_{b j}
\end{array}\right]\right.\right.
\end{aligned}
$$

$\left.\times\left[\begin{array}{l}E_{a i}+E_{a j} \\ -E_{b i} F_{j}-E_{b j} F_{i}\end{array}\right]\right)^{T}\left(P_{k}+\mu R\right)+\left(P_{k}+\mu R\right)$

$\times\left(\left[\begin{array}{ll}D_{a i}+D_{a j} & D_{b i}+D_{b j}\end{array}\right]\left[\begin{array}{cc}\Delta_{a i}+\Delta_{a j} & 0 \\ 0 & \Delta_{b i}+\Delta_{b j}\end{array}\right]\right.$

$$
\left.\left.\times\left[\begin{array}{l}
E_{a i}+E_{a j} \\
-E_{b i} F_{j}-E_{b j} F_{i}
\end{array}\right]\right)\right\}
$$

$$
\prec 0
$$

Then, based on Lemma 2, an upper bound of $\Upsilon_{1}(x, z)$ obtained as:

$$
\begin{aligned}
& \left(\frac{G_{i j}+G_{j i}}{2}\right)^{T}\left(P_{k}+\mu R\right)+\left(P_{k}+\mu R\right)\left(\frac{G_{i j}+G_{j i}}{2}\right) \\
& +\lambda^{-1}\left(P_{k}+\mu R\right)\left[D_{a i}+D_{a j} \quad D_{b i}+D_{b j}\right]\left[\begin{array}{c}
D_{a i}^{T}+D^{T}{ }_{a j} \\
D_{b i}^{T}+D^{T}{ }_{b j}
\end{array}\right] \\
& +\lambda\left[\begin{array}{l}
\left(E_{a i}+E_{a j}\right)^{T} \quad\left(-E_{b i} F_{j}-E_{b j} F_{i}\right)^{T}
\end{array}\right] \\
& \times\left[\begin{array}{l}
E_{a i}+E_{a j} \\
-E_{b i} F_{j}-E_{b j} F_{i}
\end{array}\right]\left(P_{k}+\mu R\right) \prec 0
\end{aligned}
$$

by Schur complement, we obtain,

$$
\left[\begin{array}{ccc}
\Phi_{2} & \left(P_{k}+\mu R\right)\left(D_{a i}+D_{a j}\right) & \left(P_{k}+\mu R\right)\left(D_{b i}+D_{b j}\right) \\
* & -\lambda I & 0 \\
* & * & -\lambda I
\end{array}\right] \prec 0
$$


with

$$
\begin{aligned}
\Phi_{2} & =\left(\frac{G_{i j}+G_{j i}}{2}\right)^{T}\left(P_{k}+\mu R\right)+\left(P_{k}+\mu R\right)\left(\frac{G_{i j}+G_{j i}}{2}\right) \\
& +\lambda\left(P_{k}+\mu R\right)\left[\left(E_{a i}+E_{a j}\right)^{T}\left(E_{a i}+E_{a j}\right)\right. \\
& \left.+\left(E_{b i} F_{j}+E_{b j} F_{i}\right)^{T}\left(E_{b i} F_{j}+E_{b j} F_{i}\right)^{T}\right]
\end{aligned}
$$

If (28) and (29) holds, the time derivative of the fuzzy Lyapunov function is negative. Consequently, we have $\dot{V}(x(t)) \prec 0$ and the closed loop fuzzy system (7) is stable. This is complete the proof.

\section{NUMERICAL EXAMPLE}

Consider the following T-S fuzzy system:

$$
\dot{x}(t)=\sum_{i=1}^{r} h_{i}(z(t)) A_{i} x(t)
$$

with: $r=2$

the premise functions are given by:

$$
\begin{gathered}
h_{1}\left(x_{1}(t)\right)=\frac{1+\sin x_{1}(t)}{2} ; \quad h_{2}\left(x_{1}(t)\right)=\frac{1-\sin x_{1}(t)}{2} ; \\
A_{1}=\left[\begin{array}{ll}
-5 & -4 \\
-1 & -2
\end{array}\right] ; \quad A_{2}=\left[\begin{array}{ll}
-2 & -4 \\
20 & -2
\end{array}\right] ;
\end{gathered}
$$

It is assumed that $\left|x_{1}(t)\right| \leq \frac{\pi}{2}$. For $\xi_{11}=0, \xi_{12}=0.5$, $\xi_{21}=-0.5$, and $\xi_{22}=0$, we obtain

$$
\begin{gathered}
P_{1}=\left[\begin{array}{ll}
37.7864 & 26.8058 \\
26.8058 & 36.2722
\end{array}\right] ; P_{2}=\left[\begin{array}{ll}
98.5559 & 28.7577 \\
28.7577 & 22.9286
\end{array}\right] ; \\
R=\left[\begin{array}{ll}
-1.2760 & -2.2632 \\
-2.2632 & -0.6389
\end{array}\right]
\end{gathered}
$$

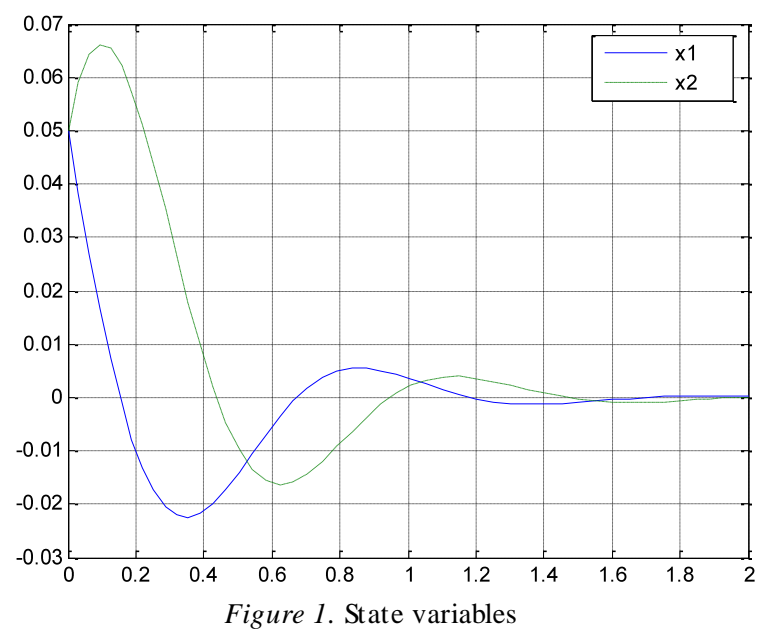

Figure 3 shows the evolution of the state variables. As can be seen, the conservatism reduction leads to very interesting results regarding fast convergence of this Takagi-Sugeno fuzzy system.

\section{CONCLUSION}

This paper provided a new condition for the stability and stabilization of Takagi-Sugeno fuzzy systems in terms of a combination of the LMI approach and the use of non-quadratic Lyapunov function as Fuzzy Lyapunov function.

In addition, a new condition of stability of uncertain system is given for Takagi-Sugeno fuzzy systems by the use of proposed fuzzy Lyapunov function.

\section{AC KNO WLEDGMENT}

The authors would like to thank the anonymous reviewers for their careful reading of this paper and for their helpful comments. This work was supported by the National High Technology Research and Development Program of China under grant no. 2006AA060101.

\section{REFERENCES}

[1] Y.Y. Cao and P.M. Frank, "Stability analysis and synthesis of nonlinear time-delay systems via Takagi-Sugeno fuzzy models", Fuzzy Sets and systems, Vol. $124 \mathrm{~N}^{\circ} 2$, pp. 213229, 2001.

[2] C. Lin, Q.G. Wang, T.H. Lee, "Delay-dependent LMI conditions for stability and stabilization of $\mathrm{T}-\mathrm{S}$ fuzzy systems with bounded time-delay", Fuzzy Sets and Systems, Vol. $157 \mathrm{~N}^{\circ}$ 9, pp. 1229-1247, 2006.

[3] L.A. Mozelli, R.M. Palhares, F.O. Souza, and E.M. Mendes, "Reducing conservativeness in recent stability conditions of TS fuzzy systems," Automatica, Vol. 45, pp. 1580-1583, 2009.

[4] C. Lin, Q.G. Wang, and T. H. Lee, "Fuzzy Weightingdependent approach to $H_{\infty}$ filter deis gn for Time-delay fuzzy systems", IEEE Transactions on Signal Processing, Vol. $55 \mathrm{~N}^{\circ} 6,2007$.

[5] C. Lin, Q.G. Wang, and T. H. Lee, LMI Approach to Analysis and Control of Takagi-Sugeno Fuzzy Systems with Time Delay, Springer-Verlag, Berlin 2007.

[6] Y.Y. Cao, and P.M. Frank. "Analysis and synthesis of nonlinear time-delay systems via fuzzy control approach". IEEE Transactions on Fuzzy Systems, 8(2), 200-211, 2000.

[7] K. Tanaka, T. Hori, and H.O. Wang, "A multiple Lyapunov function approach to stabilization of fuzzy control systems," IEEE Transactions on Fuzzy Systems, Vol. $11 \mathrm{~N}^{\circ} 4$, pp. 582-589, 2003.

[8] K. Tanaka, and H.O. Wang, Fuzzy control systems design and analysis: A linear matrix inequality approach. John Wiley and Sons, 2001.

[9] L.A. Mozelli, R.M. Palhares, F.O. Souza, and E.M. Mendes, "Reducing conservativeness in recent stability conditions of TS fuzzy systems," Automatica, Vol. 45, pp. 1580-1583, 2009.

[10] T. Takagi, and M. Sugeno, "Fuzzy identification of systems and its application to modeling and control," IEEE Trans. On System, Man and Cybernetics, vol 15 (1), pp. 116-132, 1985. 
[11] M.A.L. Thathachar, P. Viswanah, "On the Stability of Fuzzy Systems", IEEE Transactions on Fuzzy Systems, Vol. 5, Nº 1, pp. $145-151$, February 1997.

[12] K. Tanaka, T. Hori, and H.O. Wang, "A multiple Lyapunov function approach to stabilization of fuzzy control systems," IEEE Transactions on Fuzzy Systems, Vol. $11 \mathrm{~N}^{\circ} 4$, pp. 582-589, 2003.

[13] K. Tanaka, and H.O. Wang, Fuzzy control systems design and analysis: A linear matrix inequality approach. John Wiley and Sons, 2001.

[14] L. K. Wong, F.H.F. Leung, P.K.S. Tam, "Stability Design of TS Model Based Fuzzy Systems", Proceedings of the Sixth IEEE International Conference on Fuzzy Systems, Vol. 1, pp. 83-86, 1997.

[15] C.H. Fang, Y.S. Liu, S.W. Kau, L. Hong, and C.H. Lee, “A New LMI-Based Approach to Relaxed Quadratic Stabilization of T-S Fuzzy Control Systems," IEEE Transactions on Fuzzy Systems, Vol. 14, № 3, pp.386-397, June 2006.

[16] H.O. Wang, K. Tanaka, M. F. Griffin, "An Approach to Fuzzy Control of Nonlinear Systems: Stability and Design Issues”, IEEE Transactions On Fuzzy Systems, Vol. 4, ํ1, February 1996.

[17] Tanaka K., Hori T. and Wang H.O., "A fuzzy Lyapunov approach to fuzzy control system design", Proc. American Control Conf., Arlington VA, pp.4790-4795.

[18] C.W. Chen, "Stability conditions of fuzzy systems and its application to structural and mechanical systems", Advances in Engineering Software, Vol. 37, pp. 624 -629, 2006.

[19] S. Boyd, L. Ghaoui, E. Feron, V. Balakrishnan, Linear Matrix Inequalities in Systems and Control Theory, Philadelphia, PA: SIAM, 1994.

[20] Y. Manai, M. Benrejeb, "Stability for Continuous TakagiSugeno Fuzzy System based on Fuzzy Ly apunov Function', Conf. CCCA'11, Hammamet, 2011.
Yassine Manai was born in Tunisia on December 1979. He received the Master degree in Automatic and Signal Processing and the Doctorate degree in Electrical Engineering from the "Ecole Nationale d'Ingénieurs de Tunis" (ENIT) Tunisia in 2005 and 2009 respectively. His Doctorate thesis is prepared within the framework of unit research "Laboratoire de Recherche en Automatique" (LA.R.A) about Embedded System Architectures Design and Synthesis by the use of Heterogeneous Platforms.

Mohamed Benrejeb was born in Tunisia on May 1950. He received the Diploma of "Ingénieur IDN" in 1973 from the North Industrial Institute (IDN currently central school of Lille), France. In 1976, he received the engineering doctor diploma in Automatic from Technology and Science university of Lille and the doctorate es physics sciences from the same university in 1980. He is currently a full professor at the "Ecole Nationale d'Ingénieurs de Tunis" (ENIT) Tunisia and an invited Professor at the Central School of Lille. As director of the unit research "Laboratoire de Recherche en Automatique" (LA.R.A), his fields of research include system control, modelisation, analysis and synthes is of complex systems based on classical and non conventional approaches.

How to cite this paper: Yassine Manai,Mohamed Benrejeb,"New Condition of Stabilization of Uncertain Continuous Takagi-Sugeno Fuzzy System based on Fuzzy Lyapunov Function", International Journal of Intelligent Systems and Applications(IJISA), vol.4, no.4, pp.19-25, 2012. DOI: $10.5815 /$ ijisa.2012.04.03 